\title{
Algorithms for Manipulating Sequential Allocation
}

\author{
Mingyu Xiao, Jiaxing Ling \\ School of Computer Science and Engineering \\ University of Electronic Science and Technology of China \\ myxiao@gmail.com, lingjiaxing@std.uestc.edu.cn
}

\begin{abstract}
Sequential allocation is a simple and widely studied mechanism to allocate indivisible items in turns to agents according to a pre-specified picking sequence of agents. At each turn, the current agent in the picking sequence picks its most preferred item among all items having not been allocated yet. This problem is well-known to be not strategyproof, i.e., an agent may get more utility by reporting an untruthful preference ranking of items. It arises the problem: how to find the best response of an agent? It is known that this problem is polynomially solvable for only two agents and NP-complete for an arbitrary number of agents. The computational complexity of this problem with three agents was left as an open problem. In this paper, we give a novel algorithm that solves the problem in polynomial time for each fixed number of agents. We also show that an agent can always get at least half of its optimal utility by simply using its truthful preference as the response.
\end{abstract}

\section{Introduction}

Sequential allocation is a simple and widely studied mechanism to allocate indivisible items to agents (Bouveret and Lang 2011; 2014; Aziz et al. 2017). In a sequential allocation mechanism, there are several indivisible items to be allocated to some agents, each agent has a strict preference ranking over all the items, and there is a sequence of the agents, called the policy, to specify the turns of the agents to get the items. The items are allocated to the agents according to the policy: at each turn, the current agent on the policy picks the most preferred item in its preference ranking that has not yet been allocated. We give an example.

Example 1. There are five items $\{a, b, c, d, e\}$, three agents $\{1,2,3\}$ with preference rankings

$$
\begin{aligned}
& \text { Agent 1:a } \begin{array}{l}
\text { Agent 2: } 2 \succ c \succ b \succ e \succ d \succ e \\
\text { Agent } 3: e \succ b \succ d \succ c \succ a
\end{array}
\end{aligned}
$$

and a policy

$$
\pi: 13221 .
$$

Copyright (C) 2020, Association for the Advancement of Artificial Intelligence (www.aaai.org). All rights reserved.
In this example, Agent 1 will take $a$ at the first turn, Agent 3 will take $e$ at the second turn, Agent 2 will take two items $c$ and $b$ at the third and fourth turns, and Agent 1 will take $d$ at the last turn.

In sequential allocation, given a fixed policy, the outcome will only depend on the ordinal preference rankings of agents over items. It is folklore that sequential allocation is not strategyproof, which means that an agent may get more utility by reporting an untruthful preference ranking. For example, in the above instance, if Agent 1 misreports its preference ranking as $b \succ a \succ c \succ d \succ e$, then it will get items $\{b, a\}$, while originally it will get items $\{a, d\}$. Agent 1 may get more utility by taking $\{b, a\}$ since $b \succ d$. This motivates many aspects of the study on this mechanism.

There are several models based on the sequential allocation mechanism. We have different objectives to maximize the overall social welfare (Bouveret and Lang 2011) or the utility of a certain agent (Bouveret and Lang 2014), and different requirements on the pattern of the picking sequences and the number of agents. One of the earliest models studied in (Kohler and Chandrasekaran 1971) has two agents and the policy is strictly alternating $(e . g ., 121212 \ldots)$. A balanced alternation pattern of the policy (e.g., $12212112 \ldots$ ) was studied in (Brams and Taylor 2000). One interesting application of sequential allocation was found in course allocation to students and several axiomatic properties and manipulability on this application have been revealed. Budish and Cantillion (2012) investigated a randomized version of the sequential allocation mechanism to allocate courses to students, and Pareto optimal solutions for a model of course allocation were studied in (Cechlárová, Klaus, and Manlove 2018). The Boston mechanism is another sequential allocation mechanism with applications in school choice for students (Abdulkadiroglu et al. 2006; Kojima and Ünver 2014). A general and systematic study of the sequential allocation was done by Bouveret and Lang (2011) and by Kalinowski et al. (2013) from a game-theoretic view. Since the work of Kohler and Chandrasekaran (1971), a series of followup works on strategic aspects of sequential allocation have been made (Aziz, Goldberg, and Walsh 2017; Aziz, Walsh, and Xia 2015; Levine and Stange 2012; Tominaga, Todo, and Yokoo 2016; Aziz et al. 2015). 
In this paper, we consider manipulations in sequential allocation. In this model, the policy is given, and among all agents, one is the manipulator and all others are nonmanipulators. The manipulator needs to report a list of items as its preference ranking to achieve a certain objective. There are two commonly used assumptions. Firstly, the manipulator has complete information about the reported preferences of non-manipulators. This is a worst-case assumption often made in computer science and economics. Secondly, the manipulator has additive cardinal utilities for the items, although agents report strict and ordinal preferences. This assumption is standard in this research area.

We can define several problems with different objectives of the problem. The BEST RESPONSE problem is to find the best response of the manipulator (i.e., a preference ranking which allows it to obtain the maximum utility). BETTER THAN TRUTH RESPONSE is to ask whether the manipulator can get more utility than the allocation under its truthful report. AllocATION RESPONSE is to ask whether the manipulator can get a specified bundle of items. Among all these problems, BEST RESPONSE seems to be the hardest one and a solution to it can imply solutions to other problems, since other problems can be easily reduced to BEST RESPONSE. See (Aziz, Bouveret, and Lang 2017) for a recent survey on the results of these problems.

For BeSt RESPONSE, Bouveret and Lang (2011) first showed that the problem with only two agents (one manipulator and one non-manipulator) can be solved in polynomial time. Then Aziz et al. (2017) proved that it is NP-hard to compute the best response of the manipulator if the number of agents is part of the input by correcting a wrong claim in a previous paper. It becomes an open problem whether BEST RESPONSE is polynomially solvable for three or another constant number of agents (Aziz et al. 2017). This open problem is interesting because it is already known that the problem is polynomially solvable with the utility functions of the manipulator being some specified functions, such as lexi-cographic utilities and binary utilities (Bouveret and Lang 2011; Aziz et al. 2017). In this paper, we fully answer this question by giving a dynamic programming algorithm for BEST RESPONSE that runs in polynomial time for any fixed number of agents and any additive utility functions. Besides, we show that the manipulator can always get at least half of the optimal utility if it simply uses the truthful preference ranking, where the approximation ratio is tight as far as using the truthful preference ranking.

\section{Preliminaries}

In the sequential allocation problem, $m$ items are going to be allocated to $n$ agents according to a policy $\pi$, which is a sequence of agents specifying the turns of the agents to get items. The length $|\pi|$ of the policy is $m$ since there are $m$ items to be allocated. The set of items is denoted by $O=\left\{g_{1}, g_{2}, \ldots, g_{m}\right\}$ and the set of agents is denoted by $N=\{1,2, \ldots, n\}$, where Agent 1 is the manipulator and all other agents are non-manipulators. Each agent $i \in N$ has a complete preference ranking $\succ_{i}: g_{i_{1}}, g_{i_{2}}, \ldots, g_{i_{m}}$ over all items in $O$. We will write $g_{p} \succ_{i} g_{q}$ to denote that item $g_{p}$ is ranked ahead of $g_{q}$ in Agent $i$ 's preference ranking. The manipulator (Agent 1) has an additive utility function on the items $u: O \rightarrow \Re+$. For two items $g_{x}, g_{y} \in O$, it holds $u\left(g_{x}\right)>u\left(g_{y}\right)$ if and only if $g_{x} \succ_{1} g_{y}$. We use $k_{i}(i \in N)$ to denote the frequency of Agent $i$ appearing in the policy $\pi$, and use $m^{\prime}$ to denote the frequency of non-manipulators appearing in $\pi$. Then it holds that

$$
m=\sum_{i=1}^{n} k_{i}, \text { and } m^{\prime}=m-k_{1} .
$$

For BEST RESPONSE, the manipulator wants to find a picking strategy to achieve its maximum utility, i.e., a permutation of all the items, according to which to pick up items the manipulator can get the maximum utility. When we say an optimal solution to BEST RESPONSE, it is regarded as the optimal picking strategy or the bundle of items for the manipulator determined by the optimal picking strategy. We use $I=\left(O, N, \pi,\left\{\succ_{i}\right\}_{i=1}^{n}\right)$ to denote our input instance, where we omit the utility function of the manipulator to simplify the description since for most cases we only use the preference ranking $\succ_{1}$.

Once a picking strategy is given, we will get a fixed sequence of allocations of all items to agents, called allocation sequence. We will say the above picking strategy and allocation sequence are associated with each other. If there is no picking strategy associated with an allocation sequence, then the allocation sequence is called infeasible; otherwise, it is called feasible. For a feasible allocation sequence, it is easy to construct one picking strategy associated with it.

A partial allocation sequence is a subsequence of an allocation sequence beginning from the first allocation. We will use $\xi$ to denote an allocation sequence and use $\xi(i)$ to denote the partial allocation sequence of the first $i$ allocations of $\xi$. For each feasible partial allocation sequence of length $l$, there is a partial policy of length $l$ and a partial picking strategy associated with it. After executing a partial allocation sequence according to a partial policy, we will get a remaining problem which is to allocate the remaining items to the agents according to the remaining policy.

Given a (partial) allocation sequence, we say an item $g$ has been considered by Agent $i$ before the $x$ th position of the (partial) policy if during the first $x$ allocations in the sequence the last item allocated to Agent $i$ is ranked lower than item $g$ in Agent $i$ 's preference ranking. Note that an item may not be allocated to an agent even if the item has been considered by the agent.

A segment in a policy is a maximal continuous subsequence containing at most one position of a non-manipulator and only the last position of the subsequence can be the non-manipulator. A policy having $m^{\prime}$ positions of nonmanipulators can be partitioned into $m^{\prime}+1$ segments by cutting after each non-manipulator position, where the last segment is called a trivial segment. A trivial segment only contains copies of the manipulator and it may be empty (when the last position of the policy is a non-manipulator). A nontrivial segment may contain only one non-manipulator. We will use $\pi^{s}(x)$ to denote the partial policy of the first $x$ segments of $\pi$. The core of a (partial) policy is the sequence of agents obtained by deleting all occurrences of the manipula- 
tor from the (partial) policy. See Figure 1 for an illustration of the segments and cores.

Policy $\pi: 131123111$

The core: 323

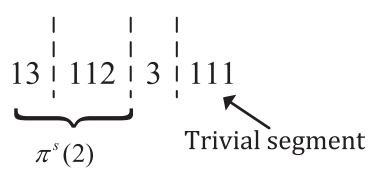

Figure 1: The segments and core

The position vector of the manipulator in a (partial) policy $\pi$ is a sequence of increasing positive integers, $\left(z_{1}, z_{2}, \ldots, z_{k_{1}}\right)$ to denote the positions of the manipulator in the policy $\pi$, i.e., the manipulator appears on the $z_{1}$ th, $z_{2}$ th, ..., and $z_{k_{1}}$ th positions in the (partial) policy $\pi$. A policy $\pi$ dominates another policy $\pi^{\prime}$ if they have the same length and the same core and it holds that $z_{i} \leq z_{i}^{\prime}$, $i \in\left\{1,2, \ldots, k_{1}\right\}$, for manipulator position vectors in $\pi$ and $\pi^{\prime}$ being $\left(z_{1}, z_{2}, \ldots, z_{k_{1}}\right)$ and $\left(z_{1}^{\prime}, z_{2}^{\prime}, \ldots, z_{k_{1}}^{\prime}\right)$, respectively. This is to say that $\pi^{\prime}$ can be obtained from $\pi$ by iteratively moving a manipulator in it to the next position. For two instances $I=\left(O, N, \pi,\left\{\succ_{i}\right\}_{i=1}^{n}\right)$ and $I^{\prime}=\left(O, N, \pi^{\prime},\left\{\succ_{i}\right.\right.$ \}$\left._{i=1}^{n}\right)$ with only different policies, if $\pi$ dominates $\pi^{\prime}$, then we say instance $I$ dominates instance $I^{\prime}$.

Our algorithm for BEST RESPONSE uses two major ideas. The first idea is to reduce instances to constrained instances, called "crucial instances". Crucial instances can be solved quickly directly. However, it is not easy to find the corresponding crucial instances and we still need to search among a large number of candidates. So we also use the second idea, which is a divide-and-conquer technique, to reduce the number of candidates. The divide-and-conquer method will split the allocation problem into two subproblems: the first one is to allocate a fixed set of items and the second one is to allocate the remaining set of items. To guarantee that we can combine optimal solutions to the two parts to construct an optimal solution for the whole problem, we need some "invariance properties". Based on "invariance properties", we can design a dynamic programming algorithm to save running time. We first introduce the two ideas in the following two sections.

\section{Crucial Instances}

In BEST RESPONSE, we may have the same optimal picking strategy for two instances with only different policies. These instances have some common properties. We will classify some instances (and their policies) that have the same optimal picking strategy and solution into a class. In each class, there is a special instance, called "crucial instance", which can be solved directly. So we will try to solve an instance by solving the corresponding crucial instance in the same class. This is the rough idea of our algorithm.

We give an example to illustrate that two instances with only different policies have the same optimal solution. In Example 1, the manipulator gets the best bundle $\{a, b\}$ by using picking strategy bacde. We use $I^{\prime}$ to denote the instance after replacing the policy $\pi$ : 13221 with policy $\pi^{\prime}: 32121$. In $I^{\prime}$, the manipulator can get the same best bundle $\{a, b\}$ by using the same picking strategy. Compared with $\pi$, the manipulator has a lower priority to pick items in $\pi^{\prime}$. However, the manipulator still can get the optimal solution. Note that, at the first position of the policy $\pi$, the manipulator picks an item that will not be considered by any non-manipulator before the 3rd allocation. So we can delay the allocation of $b$ to Agent 1 from position 1 to position 3 without changing the optimality. Given an instance, we want to know how much we can delay the positions of the manipulator without losing the optimality and the "worst" policy will be "crucial".

Definition 1 (Crucial Instance). For an instance $I=$ $\left(O, N, \pi,\left\{\succ_{i}\right\}_{i=1}^{n}\right)$, if for any policy $\pi^{\prime} \neq \pi$ dominated by $\pi$, the optimal solution to the dominated instance $I^{\prime}=$ $\left(O, N, \pi^{\prime},\left\{\succ_{i}\right\}_{i=1}^{n}\right)$ is worse than that to $I$, then we say $I$ is a crucial instance. Let $I^{\prime}=\left(O, N, \pi^{\prime},\left\{\succ_{i}\right\}_{i=1}^{n}\right)$ be a crucial instance, for any instance $I=\left(O, N, \pi,\left\{\succ_{i}\right\}_{i=1}^{n}\right)$ dominating $I^{\prime}$, we say $I^{\prime}$ is a corresponding crucial instance to $I$.

A corresponding crucial instance of an instance may be itself when it is already a crucial instance. We show some properties of crucial instances.

Lemma 1. Given two instances $I=\left(O, N, \pi,\left\{\succ_{i}\right\}_{i=1}^{n}\right)$ and $I^{\prime}=\left(O, N, \pi^{\prime},\left\{\succ_{i}\right\}_{i=1}^{n}\right)$, where $I$ dominates $I^{\prime}$. By using the same picking strategy, the manipulator in instance $I$ will get a bundle with total utility not less than that in $I^{\prime}$. Furthermore, for any picking strategy $S^{\prime}$, there is a picking strategy $S$ such that by using $S$ in I the manipulator can get the same bundle as that by using $S^{\prime}$ in $I^{\prime}$.

Proof. The first claim is easy to observe. We focus on the second claim.

We define the picking strategy $S$ to $I$ as follows: order the items according to the ordering of allocations to the manipulator in $I^{\prime}$ by using $S^{\prime}$, i.e., an item is ranked on the $i$ th position in $S$ if it is the $i$ th item allocated to the manipulator in $I^{\prime}$, and all other items not allocated to the manipulator in $I^{\prime}$ are listed behind with any order. Let $\left(z_{1}, z_{2}, \ldots, z_{k_{1}}\right)$ and $\left(z_{1}^{\prime}, z_{2}^{\prime}, \ldots, z_{k_{1}}^{\prime}\right)$ be the position vectors of the manipulator of $\pi$ and $\pi^{\prime}$. Since $\pi$ dominates $\pi^{\prime}$, we know that $z_{i} \leq z_{i}^{\prime}$ for any $i \in\left\{1,2, \ldots, k_{1}\right\}$. If an item can be allocated to the manipulator at position $z_{i}^{\prime}$ in $\pi^{\prime}$, then it can also be allocated to the manipulator at position $z_{i} \leq z_{i}^{\prime}$ in $\pi$, since only a subset of items have been allocated before position $z_{i}$ in $\pi$ (compared to the situation at position $z_{i}^{\prime}$ in $\pi^{\prime}$ ). So at each position, the manipulator can always get the current item on its picking strategy $S$. By using $S$, the manipulator in $I$ gets the same bundle as that by using $S^{\prime}$ in $I^{\prime}$.

Corollary 1. Let $I=\left(O, N, \pi,\left\{\succ_{i}\right\}_{i=1}^{n}\right)$ be an instance and $I^{\prime}=\left(O, N, \pi^{\prime},\left\{\succ_{i}\right\}_{i=1}^{n}\right)$ be a corresponding crucial instance. The optimal solutions to $I$ and $I^{\prime}$ have the same total utility. Furthermore, given an optimal picking strategy for $I^{\prime}$, an optimal picking strategy for I can be constructed in polynomial time.

Proof. By Lemma 1, we know that the optimal solution to $I$ is not worse than that to $I^{\prime}$. On the other hand, by the definition of crucial instances, we know that the optimal solution to $I^{\prime}$ is not worse than that to $I$. So $I$ and $I^{\prime}$ will have the 
same total utility of the optimal solutions. In the proof of Lemma 1, a method to construct the optimal picking strategy for $I$ is already given.

Our idea is that to solve an instance, we turn to solve a corresponding crucial instance. Next, we first introduce our algorithm for crucial instances. The algorithm is a greedy algorithm, called GreedyAlg.

Algorithm GreedyAlg The algorithm GreedyAlg takes a (sub) instance of BEST RESPONSE as the input, and outputs an allocation sequence with the corresponding picking strategy for the manipulator. However, the output allocation sequence may not be optimal for non-crucial instances.

The main idea of the algorithm is as follows. We allocate items to agents according to the policy. Assume that we have allocated the first $i-1$ items to agents in the input instance $I=\left(O, N, \pi,\left\{\succ_{i}\right\}_{i=1}^{n}\right)$. If the $i$ th position in the policy $\pi$ is a non-manipulator, we let the non-manipulator pick its most preferred item that has not yet been allocated. Next, we consider the situation that the $i$ th position in the policy $\pi$ is the manipulator. The algorithm decides the item that should be assigned to the manipulator at the $i$ th turn by the following method.

We let $I^{\prime}=\left(O^{\prime}, N, \pi^{\prime},\left\{\succ_{i}^{\prime}\right\}_{i=1}^{n}\right)$ be the remaining instance after allocating the first $i-1$ items in $I$. Then the first position in $\pi^{\prime}$ is the manipulator. Let $\pi_{-1}$ be the core of $\pi^{\prime}$. If $\pi_{-1}$ is empty, we assign the best remaining item $o$ in the truthful preference ranking of the manipulator to the manipulator at the $i$ th position of $\pi$ and let $o$ be the $i$ th object in the picking strategy of the manipulator. If $\pi_{-1}$ is not empty, we let $o_{f}$ be the first item allocated to an agent when simulating $\pi_{-1}$ on $\left(O^{\prime}, N \backslash\{1\}, \pi_{-1},\left\{\succ_{i}^{\prime}\right\}_{i=1}^{n}\right)$, i.e., the favourite item in $O^{\prime}$ of the first agent in $\pi_{-1}$. GreedyAlg will assign item $o_{f}$ to the manipulator at the $i$ th position of $\pi$ and let $o_{f}$ be the $i$ th object in the picking strategy of the manipulator.

According to the above method, the algorithm decides the items to be assigned to the manipulator from the first occurrence of 1 to the last occurrence of 1 in $\pi$ and then we can get a full allocation sequence and a picking strategy for the manipulator. This is the algorithm GreedyAlg.

Lemma 2. The greedy algorithm GreedyAlg can find optimal solutions to crucial instances.

Proof. Assume to the contrary that a solution found by GreedyAlg is not optimal for a crucial instance. Then at least on one position, say the $i$ th position of $\pi$, the manipulator does not pick item $o_{f}$ defined above (the next item will be taken by a non-manipulator in $\left(O^{\prime}, N \backslash\{1\}, \pi_{-1},\left\{\succ_{i}^{\prime}\right\}_{i=1}^{n}\right)$ with $\left.\pi_{-1}\right)$. we can move the $i$ th position of 1 to the behind of the first non-manipulator in $\pi$ to get a dominated instance $I^{\prime}$, where $I^{\prime}$ has the same optimal solution as $I$, which contradicts the fact that $I$ is a crucial instance.

By using the priority queue, GreedyAlg can be implemented in linear time. We can also see that the picking strategy returned by GreedyAlg for each instance is unique. The allocation sequence returned by GreedyAlg on a (sub) instance is called greedy. Given an allocation sequence, we can easily check whether it is greedy or not. The concept of greedy allocation sequences is also important and will be used later.

Although crucial instances can be solved quickly, it is still hard to find a corresponding crucial instance for an arbitrary instance. We need to reveal more properties for dominated instances.

Lemma 1 implies that the optimal solution to an instance is not worse than the optimal solution to any dominated instance. Clearly, the opposite direction of Lemma 1 may not hold. We prove the following lemma.

Lemma 3. Let I be an instance and $\mathrm{P}$ be the set of instances dominated by $I$. For each $I^{\prime} \in \mathrm{P}$, we use $G\left(I^{\prime}\right)$ to denote the greedy allocation sequence of $I^{\prime}$. Assume that the greedy allocation sequence $G\left(I_{0}\right)\left(I_{0} \in \mathrm{P}\right)$ gets the best solution among all $G\left(I^{\prime}\right)$ with $I^{\prime} \in \mathrm{P}$. Then $I_{0}$ is a crucial instance corresponding to $I$.

The correctness of this lemma follows from Lemma 1, Corollary 1 and Lemma 2. Lemma 1 says any dominated instance $I^{\prime}$ will not have a better solution than $I$. Corollary 1 says that there is at least one dominated instance, the corresponding crucial instance will achieve the same optimal solution to $I$. The greedy allocation sequence may not be optimal for any instance. But it is optimal for a crucial instance by Lemma 2 . Therefore, among all the greedy solutions, the best one is for a corresponding crucial instance.

Lemma 3 implies that we can solve BEST RESPONSE by taking each dominated instance as a candidate for a corresponding crucial instance and use GreedyAlg to solve it. We analyze the running time of this algorithm. Let $\left(z_{1}, z_{2}, \ldots, z_{k_{1}}\right)$ and $\left(z_{1}^{\prime}, z_{2}^{\prime}, \ldots, z_{k_{1}}^{\prime}\right)$ be the position vectors of the manipulator of two policies $\pi$ and $\pi^{\prime}$. We know that $\pi$ dominates $\pi^{\prime}$ if and only if $z_{i} \leq z_{i}^{\prime}$ holds for any $i \in\left\{1,2, \ldots, k_{1}\right\}$. The length of these policies is $m$. So for $i \in\left\{1,2, \ldots, k_{1}\right\}$, the value of $z_{i}^{\prime}$ can be any integer between $\max \left\{z_{i}, z_{i-1}^{\prime}+1\right\}$ and $m$. Combinatorial analyses with some relaxations can easily establish an upper bound of $O\left(\mathrm{~m}^{k_{1}}\right)$ for the number of dominated policies. The algorithm to consider all dominated instances is not polynomial when the frequency $k_{1}$ of the manipulator in the policy is not a constant.

We will use a dynamic programming technique to reduce the number of dominated instances to a polynomial without losing an optimal solution. To do so, we need the following properties.

\section{Invariance Properties}

Our idea is a divide-and-conquer method. We will partition the problem into two subproblems, the first part is to allocate the first $i$ items and the second part is to allocate the remaining items. We need to find the properties in the first part that keep the invariance of the second part. Once we find these properties, we may only need to find the optimal allocation sequence of the first part for the manipulator satisfying these properties (for each fixed allocation sequence for the second part). In this way, we may be able to use dynamic programming to reduce redundant cases without losing an optimal solution. 
It is easy to verify that the remaining problems are the same after executing two partial allocation sequences satisfying the following two conditions:

1. The number of items allocated to each agent (including the manipulator) is the same;

2. The set of items allocated to all the agents is the same.

However, it is still hard to find all partial allocation sequences satisfying the above two conditions. In order to get a polynomial-time algorithm, we add the third condition below

3. The last item allocated to each non-manipulator is the same.

Definition 2 (Invariance Relation). Two (partial) allocation sequences are in the invariance relation if they satisfy the above three conditions.

Recall that for an allocation sequence $\xi$, we use $\xi(i)$ to denote the partial allocation sequence of the first $i$ allocations.

Lemma 4. Let $\xi$ be a feasible allocation sequence and $\xi(i)$ $(1 \leq i \leq m)$ be a partial allocation sequence. Let $\xi^{\prime}(i)$ be another partial allocation sequence that is in the invariance relation with $\xi(i)$. The allocation sequence $\xi^{\prime}$ obtained by replacing $\xi(i)$ with $\xi^{\prime}(i)$ in $\xi$ is still a feasible allocation sequence.

Proof. According to the definition of the invariance relation, we know that in $\xi(i)$ and $\xi^{\prime}(i)$ the same set of items are allocated, and the remaining problems after executing them are the same. Therefore we can exchange $\xi(i)$ and $\xi^{\prime}(i)$ in larger allocation sequences.

The divide-and-conquer idea based on Lemma 4 will be embedded in our dynamic programming algorithm. We will see that the algorithm will only split the problem between segments.

\section{The Dynamic Programming Algorithm}

Equipped with the above properties, we are ready to describe the dynamic programming algorithm. The main idea of the algorithm is still based on Lemma 3. However, we will use Lemma 4 to reduce the number of subproblems.

Recall that $m^{\prime}$ is the number of non-manipulator positions in the policy $\pi$. For any integer $1 \leq x \leq m^{\prime}$, let $k(x)$ denote the times of the manipulator appearing during the first $x$ segments of the policy $\pi$, i.e., the period from the beginning of $\pi$ to the $x$ th position of a non-manipulator. For any dominated instance $I^{\prime}$, the occurrences of the manipulator in the first $x$ segments of the policy in $I^{\prime}$ is at most $k(x)$. Recall that $\pi^{s}(x)$ is the partial policy of the first $x$ segments of $\pi$.

Note that any (partial) allocation sequence determines a (partial) policy, which is a sequence of the agents according to the sequence of allocations. We say the (partial) policy is associated with the (partial) allocation sequence.

We use $\operatorname{pro}\left(x, y, i_{2}, \ldots, i_{n}\right)$ to denote the set of all feasible partial allocation sequences satisfying the following conditions:

1. The core of the partial policy associated with the partial allocation sequence is the same as the core of $\pi^{s}(x)$;
2. The last allocation in the partial allocation sequence is to allocate an item to a non-manipulator;

3. Exactly $x$ items are allocated to non-manipulators and exactly $y$ items are allocated to the manipulator;

4. For each $j \in\{2,3, \ldots, n\}$, the last item allocated to Agent $j$ is the $i_{j}$ th item in its preference ranking, where $i_{j}$ can be 0 which means no item is allocated to Agent $j$;

5. For each $r \in\{1,2, \ldots, x\}$, during the first $r$ segments of the associated partial policy at most $k(r)$ items are allocated to the manipulator;

6. The partial allocation sequence is a greedy one.

The domains of the parameters in $\operatorname{pro}\left(x, y, i_{2}, \ldots, i_{n}\right)$ are as follows: $x \in\left\{0,1, \ldots, m^{\prime}\right\}, y \in\left\{0,1, \ldots, k_{1}\right\}$ and $i_{2}, i_{3}, \ldots, i_{n} \in\{0,1, \ldots, m\}$. We may not describe the domains of the parameters when they are clear from the context.

Note that even all of $x, y$ and $i_{j}$ are fixed, the set pro $\left(x, y, i_{2}, \ldots, i_{n}\right)$ may contain several different allocation sequences, because the definition does not fix the positions of the $y$ manipulators in the corresponding (partial) policy. We have the following property.

Lemma 5. Any two partial allocation sequences in pro $\left(x, y, i_{2}, \ldots, i_{n}\right)$ are in the invariance relation.

Lemma 5 can be proved by checking each of the three conditions of the invariance relation one by one, which is not hard and omitted here due to the limited space.

We use $\operatorname{opt}\left(x, y, i_{2}, \ldots, i_{n}\right)$ to denote a partial allocation sequence in $\operatorname{pro}\left(x, y, i_{2}, \ldots, i_{n}\right)$ where the manipulator gets the optimal solution. Note that $\operatorname{pro}\left(x, y, i_{2}, \ldots, i_{n}\right)$ is possible to be empty and for this case we let $\operatorname{opt}\left(x, y, i_{2}, \ldots, i_{n}\right)=\perp$.

The allocation sequence $\operatorname{opt}\left(x, y, i_{2}, \ldots, i_{n}\right)$ even for $x=m^{\prime}$ may not be a complete allocation sequence of length $m$, since $y$ may be smaller than $k_{1}$ and some allocations to the manipulator are still left. In fact, $\operatorname{opt}(x=$ $\left.m^{\prime}, y, i_{2}, \ldots, i_{n}\right)$ is a partial allocation sequence only missing the last part of the allocations corresponding to the trivial segment of the policy. We use $\operatorname{opt}^{*}\left(x=m^{\prime}, y, i_{2}, \ldots, i_{n}\right)$ to denote the complete allocation sequence obtained from $\operatorname{opt}\left(x=m^{\prime}, y, i_{2}, \ldots, i_{n}\right)$ plus the $k_{1}-y$ allocations of the $k_{1}-y$ best remaining items to the manipulator.

The following two lemmas will say that the best allocation sequence among all $o p t^{*}\left(x, y, i_{2}, \ldots, i_{n}\right)$ with $x=m^{\prime}$ will lead to the optimal solution to the original instance.

Lemma 6. For any $y \in\left\{0,1, \ldots, k_{1}\right\}$ and $i_{2}, i_{3}, \ldots, i_{n} \in$ $\{0,1, \ldots, m\}$, if $\operatorname{opt}\left(x=m^{\prime}, y, i_{2}, \ldots, i_{n}\right) \neq \perp$, then opt $^{*}\left(x=m^{\prime}, y, i_{2}, \ldots, i_{n}\right)$ is a greedy allocation sequence for an instance dominated by the original instance.

Proof. By the definition, we know that $\operatorname{opt}(x=$ $\left.m^{\prime}, y, i_{2}, \ldots, i_{n}\right)$ is a greedy partial allocation sequence. Since $o p t^{*}\left(x=m^{\prime}, y, i_{2}, \ldots, i_{n}\right)$ is obtained from opt $(x=$ $\left.m^{\prime}, y, i_{2}, \ldots, i_{n}\right)$ by adding behind $k_{1}-y$ best allocations to the manipulator, we know that $\operatorname{opt}^{*}\left(x=m^{\prime}, y, i_{2}, \ldots, i_{n}\right)$ is also greedy. We consider the policy $\pi^{*}$ corresponding to the greedy allocation sequence $o p t^{*}\left(x=m^{\prime}, k_{1}-z, i_{2}, \ldots, i_{n}\right)$. By the 5 th item in the definition of $\operatorname{pro}\left(x, y, i_{2}, \ldots, i_{n}\right)$, we 
know that for each $r \in\{1,2, \ldots, x\}$, during the first $r$ segments at most $k(r)$ items are allocated to the manipulator. This means $\pi^{*}$ is dominated by the original policy $\pi$.

Lemma 7. Let $I_{c}$ be a crucial instance corresponding to $I$, where the trivial segment in $I_{c}$ consists of $z$ occurrences of the manipulator $\left(0 \leq z \leq k_{1}\right)$. Assume that in the optimal solution to $I_{c}$, for each $\bar{j} \in\{2,3, \ldots, n\}$, the last item allocated to Agent $j$ is the $a_{j}$ th item in its preference ranking. Then opt* $\left(m^{\prime}, k_{1}-z, a_{2}, \ldots, a_{n}\right)$ leads to the optimal solution to the original instance $I$.

Proof. The greedy allocation sequence $S$ to $I_{c}$, also leading to an optimal solution to the original instance $I$, is a candidate for $o p t^{*}\left(m^{\prime}, y, a_{2}, \ldots, a_{n}\right)$. On the other hand, by Lemmas 6 and 1, we know that $o p t^{*}\left(m^{\prime}, k_{1}-z, a_{2}, \ldots, a_{n}\right)$ is not better than $S$. Since $\operatorname{opt}^{*}\left(\mathrm{~m}^{\prime}, k_{1}-z, a_{2}, \ldots, a_{n}\right)$ is chosen as the best one, we know that $o p t^{*}\left(m^{\prime}, k_{1}-\right.$ $\left.z, a_{2}, \ldots, a_{n}\right)$ is as good as $S$.

We can not directly compute an optimal solution to the original instance $I$ according to Lemma 7, since we do not know the values of $a_{j}$ in Lemma 7. However, by Lemma 3, Lemma 6 and Lemma 7, we know that the best one among all $o p t^{*}\left(x, y, i_{2}, \ldots, i_{n}\right)$ with $x=m^{\prime}$ will get an optimal solution to the original instance $I$. So our algorithm contains the following three main steps.

1 Compute all opt $\left(x, y, i_{2}, \ldots, i_{n}\right)$ by calling the subalgorithm OPT;

2 Compute all $\operatorname{opt}^{*}\left(x, y, i_{2}, \ldots, i_{n}\right)$ with $x=m^{\prime}$ from $\operatorname{opt}\left(m^{\prime}, y, i_{2}, \ldots, i_{n}\right)$;

3 Find the best one among all $\operatorname{opt}^{*}\left(x, y, i_{2}, \ldots, i_{n}\right)$ with $x=m^{\prime}$.

The sub algorithm OPT in Step 1 is a dynamic programming algorithm that compute all opt $\left(x, y, i_{2}, \ldots, i_{n}\right)$ in an order with a nonincreasing value of $x$. Before presenting the whole procedure of OPT, we introduce the idea in the algorithm.

Assume that all $\operatorname{opt}\left(x^{\prime}, y, i_{2}, \ldots, i_{n}\right)$ for $x^{\prime}<x$ have been computed. We use the following idea to compute $\operatorname{opt}\left(x, y, i_{2}, \ldots, i_{n}\right)$.

We use $r$ to denote the non-manipulator on $x$ th position of the core of $\pi$, i.e., the $x$ th non-manipulator in $\pi$ is Agent $r$. Assume that $\operatorname{opt}\left(x, y, i_{2}, \ldots, i_{n}\right) \neq \perp$. Let $\pi_{x}$ be the policy corresponding to opt $\left(x, y, i_{2}, \ldots, i_{n}\right)$. We further assume that the last segment of $\pi_{x}$ consists of $q$ occurrences of the manipulator and one occurrence of Agent $r$. Then opt $\left(x, y, i_{2}, \ldots, i_{n}\right)$ is given by the allocations $L_{1}$ of items to the first $x-1$ segments of $\pi_{x}$ plus the allocations $L_{2}$ of items to the last segment of $\pi_{x}$.

Since we require that the allocation sequence in $\operatorname{opt}\left(x, y, i_{2}, \ldots, i_{n}\right)$ is greedy, we know that $L_{2}$ is given by $q$ allocations of the first $q$ remaining items on Agent $r$ 's preference ranking to the manipulator plus one allocation of the $(q+1)$ th remaining item to Agent $r$. Furthermore, the last item allocated to Agent $r$ must be the $i_{r}$ th item in Agent $r$ 's preference ranking. By Lemma 4, Lemma 5 and the fact that the utility function is additive, we know that $L_{1}$ is given by opt $\left(x-1, y-q, i_{2}, \ldots, i_{r}^{*}, \ldots, i_{n}\right)$ for some $i_{r}^{\prime} \leq i_{r}-(q+1)$.
However, we do not know the value of $q$ and $i_{r}^{*}$. In the algorithm, we try all possible values for $q$ and $i_{r}^{*}$. Lemma 3 can guarantee that the best one among them is the correct allocation sequence we are seeking for. The whole procedure of OPT is presented in Algorithm 1.

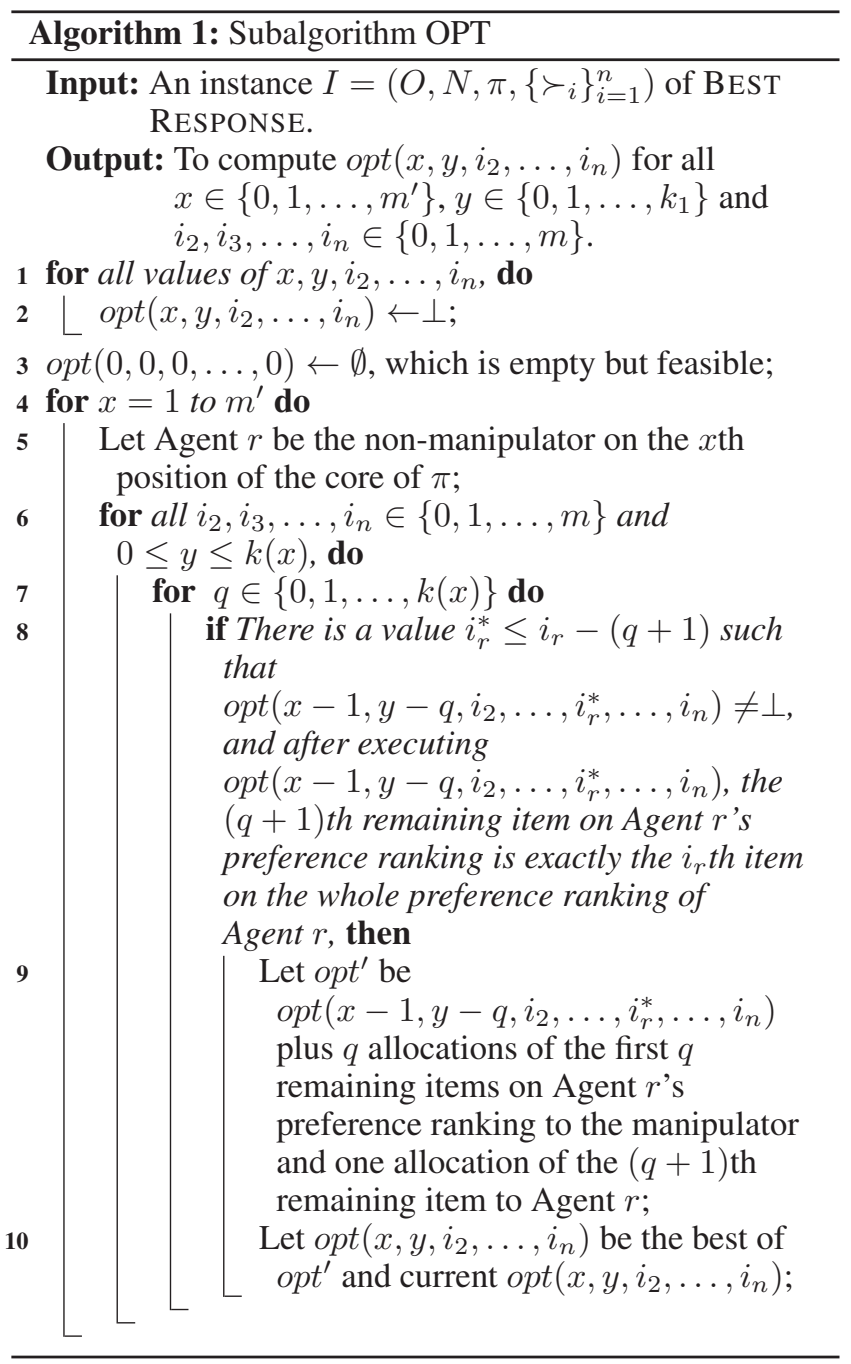

Next, we analyze the running time of the whole algorithm. The algorithm contains three steps.

The first step is to compute $\operatorname{opt}\left(x, y, i_{2}, \ldots, i_{n}\right)$ for $i_{2}, i_{3}, \ldots, i_{n} \in\{0,1, \ldots, m\}, x \in\left\{0,1, \ldots, m^{\prime}=m-k_{1}\right\}$ and $y \in\left\{0,1, \ldots, k_{1}\right\}$. In total, there are $(1+m)^{n-1}(m-$ $\left.k_{1}+1\right)\left(k_{1}+1\right)<(1+m)^{n+1}$ subproblems need to be solved. For each subproblem opt $\left(x, y, i_{2}, \ldots, i_{n}\right)$ with $x>0$, we compute them from Steps 3 to 9 in OPT. In Step 6 , we have $k(x)+1$ loops. For each loop, we may use at most $O\left(i_{t} m\right)$ time. So for each subproblem $\operatorname{opt}\left(x, y, i_{2}, \ldots, i_{n}\right)$, our algorithm uses at most $O\left(\mathrm{~m}^{3}\right)$ time. OPT runs in $O((1+$ $m)^{n+4}$ ) time.

Step 2 takes at most $O\left((1+m)^{n+2}\right)$ time to extend all $\operatorname{opt}\left(x=m^{\prime}, y, i_{2}, \ldots, i_{n}\right)$ to opt $\left(x=m^{\prime}, y, i_{2}, \ldots, i_{n}\right)$.

Step 3 is to find the best one among all opt* $(x=$ $\left.m^{\prime}, y, i_{2}, \ldots, i_{n}\right)$, which can be done in $O\left((1+m)^{n+1}\right)$ 
time.

Theorem 1. BEST RESPONSE can be solved in $O((1+$ $m)^{n+4}$ ) time.

For each constant number $n$ of agents, BEST RESPONSE is polynomially solvable.

\section{A 0.5-Approximation Algorithm}

Although for each fixed number of agents, the manipulating sequential allocation problem can be solved in polynomial time, the running time is exponential in the number $n$ of agents. When $n$ is large, the algorithm will still be slow. So we also consider approximation algorithms for the problem. We prove that

Theorem 2. For an instance of BEST RESPONSE with additive utility functions, if the manipulator takes the truthful preference ranking as its picking strategy, it can get a bundle with the total utility being at least half of that of the optimal solution.

Proof. Let $I^{\prime}$ be the corresponding crucial instance of the input instance $I$. By Corollary 1, we know that an optimal solution to $I^{\prime}$ is an optimal solution to $I$. By Lemma 1 , we also know that a solution to $I$ is at least as good as that to $I^{\prime}$ under the same picking strategy. So we only need to prove the theorem holds for crucial instance $I^{\prime}$ and next we assume that the input instance is crucial.

We use $\xi_{A}$ and $\xi_{B}$ to denote the allocations by taking the optimal picking strategy and by taking the truthful preference as the picking strategy, respectively. Let $A=$ $\left\{a_{1}, a_{2}, \ldots, a_{k_{1}}\right\}$ be the bundle obtained by $\xi_{A}$ and $B=$ $\left\{b_{1}, b_{2}, \ldots, b_{k_{1}}\right\}$ be the bundle obtained by $\xi_{B}$, where we assume that the items in the above two sets are listed according to the picking order.

We first prove that for any index $1 \leq i \leq k_{1}$ such that $a_{i} \succ_{1} b_{i}$, the item $a_{i}$ is also in $B$. Assume to the contrary that $a_{i} \notin B$, which means that item $a_{i}$ is not taken into the solution in $\xi_{B}$. The allocation of item $a_{i}$ in $\xi_{A}$ and the allocation of item $b_{i}$ in $\xi_{B}$ happen as the same position of the policy, say the $x$ th position. In $\xi_{B}$, an item $b_{i}$ with $u\left(b_{i}\right)<u\left(a_{i}\right)$ is allocated at the $x$ th position, which means that $a_{i}$ has already been allocated to some agent before the $x$ th position in $\xi_{B}$ as the picking strategy in $\xi_{B}$ is the truthful preference. However, the instance is a crucial instance and the optimal allocation sequence in $\xi_{A}$ is greedy. Item $a_{i}$ is impossible to be allocated to a non-manipulator before the $x$ th position in $\xi_{B}$. Then $a_{i}$ can only be allocated to the manipulator in $\xi_{B}$, which is a contradiction to the assumption that $a_{i} \notin B$. So the above claim holds.

Let $L=\left\{i_{1}, i_{2}, \ldots, i_{l}\right\}$ be the set of indices $i_{j}$ such that $a_{i_{j}} \succ_{1} b_{i_{j}}$. Let $\bar{L}=\left\{1,2, \ldots, k_{1}\right\} \backslash L$. Note that $\bar{L}$ is not empty and index 1 is always in $\bar{L}$. By the above claim, we have that

$$
\sum_{i \in L} u\left(a_{i}\right)<\sum_{b \in B} u(b) .
$$

By the definitions of $L$ and $\bar{L}$, we have that

$$
\sum_{i \in \bar{L}} u\left(a_{i}\right) \leq \sum_{i \in \bar{L}} u\left(b_{i}\right) \leq \sum_{b \in B} u(b) .
$$

By summing up the above two inequalities, we get that

$$
\sum_{a \in A} u(a)<2 \sum_{b \in B} u(b) .
$$

We also give a simple example, where the approximation ratio cannot be $0.5+\epsilon^{\prime}$ for any constant $\epsilon^{\prime}>0$. This will show the approximation ratio of 0.5 is tight for the mechanism of using the truthful preference. There are three items $O=\left\{g_{1}, g_{2}, g_{3}\right\}$ to be allocated to two agents $N=\{1,2\}$. The preference rankings are $\succ_{1}: g_{1}, g_{2}, g_{3}$ and $\succ_{2}: g_{2}, g_{3}, g_{1}$. The policy is $\pi: 121$. The utility function of the manipulator is that $u\left(g_{1}\right)=1, u\left(g_{2}\right)=1-\epsilon$ and $u\left(g_{3}\right)=\epsilon$. If the manipulator use the picking strategy $g_{2} g_{1} g_{3}$, it can get items $g_{2}$ and $g_{1}$ with the utility $2-\epsilon$. If the manipulator use the truthful preference ranking $g_{1} g_{2} g_{3}$ as the picking strategy, it can only get items $g_{1}$ and $g_{3}$ with the utility $1+\epsilon$. The approximation ratio is $\frac{1+\epsilon}{2-\epsilon}=0.5+\frac{1.5 \epsilon}{2-\epsilon}$, where $\frac{1.5 \epsilon}{2-\epsilon}$ can be arbitrarily small.

\section{Conclusion}

BEST RESPONSE can be regarded as one of the hardest natural problems in manipulating sequential allocation problems, since most other problems can be reduced to it. It has been known for years that BEST RESPONSE with only two agents can be solved in polynomial time. However, it took more effort to establish the NP-hardness of BEST RESPONSE with an unbounded number of agents. In this paper, we complete the "gap" by showing that BEST RESPONSE is polynomially solvable for any constant number of agents. Furthermore, we show that we can always get a 0.5 -approximation solution if taking the truthful preference ranking of the manipulator as its picking strategy. Furthermore, the ratio 0.5 is tight as far as using the truthful preference ranking. It may be interesting to consider the approximation ratio for using the truthful response on more no-strategyproof problems.

\section{Acknowledgments}

The work is supported by the National Natural Science Foundation of China, under grants 61972070 and 61802049.

\section{References}

Abdulkadiroglu, A.; Pathak, P.; Roth, A. E.; and Sonmez, T. 2006. Changing the boston school choice mechanism. Technical report, National Bureau of Economic Research.

Aziz, H.; Gaspers, S.; Mackenzie, S.; Mattei, N.; Narodytska, N.; and Walsh, T. 2015. Manipulating the probabilistic serial rule. In Proceedings of the 2015 International Conference on Autonomous Agents and Multiagent Systems, AAMAS 2015, Istanbul, Turkey, May 4-8, 2015, 1451-1459.

Aziz, H.; Bouveret, S.; Lang, J.; and Mackenzie, S. 2017. Complexity of manipulating sequential allocation. In Proceedings of the Thirty-First AAAI Conference on Artificial Intelligence, February 4-9, 2017, San Francisco, California, USA., 328-334. 
Aziz, H.; Bouveret, S.; and Lang, J. 2017. Manipulating sequential allocation: an overview. Online manuscript.

Aziz, H.; Goldberg, P.; and Walsh, T. 2017. Equilibria in sequential allocation. In Algorithmic Decision Theory - 5th International Conference, ADT 2017, Luxembourg, Luxembourg, October 25-27, 2017, Proceedings, 270-283.

Aziz, H.; Walsh, T.; and Xia, L. 2015. Possible and necessary allocations via sequential mechanisms. In Proceedings of the Twenty-Fourth International Joint Conference on Artificial Intelligence, IJCAI 2015, Buenos Aires, Argentina, July 25-31, 2015, 468-474.

Bouveret, S., and Lang, J. 2011. A general elicitation-free protocol for allocating indivisible goods. In IJCAI 2011, Proceedings of the 22nd International Joint Conference on Artificial Intelligence, Barcelona, Catalonia, Spain, July 1622, 2011, 73-78.

Bouveret, S., and Lang, J. 2014. Manipulating picking sequences. In ECAI 2014 - 21st European Conference on Artificial Intelligence, 18-22 August 2014, Prague, Czech Republic - Including Prestigious Applications of Intelligent Systems (PAIS 2014), 141-146.

Brams, S. J., and Taylor, A. D. 2000. The win-win solution - guaranteeing fair shares to everybody. W. W. Norton \& Company.

Budish, E., and Cantillon, E. 2012. The multi-unit assignment problem: Theory and evidence from course allocation at harvard. THE AMERICAN ECONOMIC REVIEW 102(5):2237-2271.

Cechlárová, K.; Klaus, B.; and Manlove, D. F. 2018. Pareto optimal matchings of students to courses in the presence of prerequisites. Discrete Optimization 29:174-195.

Kalinowski, T.; Narodytska, N.; and Walsh, T. 2013. A social welfare optimal sequential allocation procedure. In $I J$ CAI 2013, Proceedings of the 23rd International Joint Conference on Artificial Intelligence, Beijing, China, August 3-9, 2013, 227-233.

Kohler, D. A., and Chandrasekaran, R. 1971. A class of sequential games. Operations Research 19(2):270-277.

Kojima, F., and Ünver, M. U. 2014. The "boston" schoolchoice mechanism: an axiomatic approach. Economic Theory 55(3):515-544.

Levine, L., and Stange, K. E. 2012. How to make the most of a shared meal: Plan the last bite first. The American Mathematical Monthly 119(7):550-565.

Tominaga, Y.; Todo, T.; and Yokoo, M. 2016. Manipulations in two-agent sequential allocation with random sequences. In Proceedings of the 2016 International Conference on Autonomous Agents \& Multiagent Systems, Singapore, May 913, 2016, 141-149. 